\title{
Infliximab for Crohn's disease: More questions than answers
}

\author{
Hugh James Freeman MD CM FRCPC FACP FACG
}

I nfliximab (formerly cA2, Centocor, Inc, USA) is a biological agent that was first reported for the treatment of Crohn's disease in 1995 (1). It is a chimeric mouse-human immunoglobulin G1 monoclonal antibody to tumour necrosis factor alpha (TNF- $\alpha$ ), which is thought to play an important role as a mediator of inflammation in Crohn's disease. Although it neutralizes TNF, antibody infusions also cause other immunological effects that are less well defined, including changes in cell lysis through complement fixation or antibody-dependent cell-mediated cytotoxicity $(2,3)$. Indeed, in Crohn's disease tissues, intestinal mucosal cells have been shown to express more TNF (as well as other inflammatory mediators) than healthy individuals (4-6). TNF may regulate some components of the immune response, including those associated with intestinal inflammation; however, the precise mechanism in Crohn's disease has not been fully defined and may be quite complex. There are data from controlled trials to support its use as an intravenous infusion therapy to control symptoms in some patients with Crohn's disease (7-9). Despite media reports, it is not a 'cure' for the disease. Infliximab received fasttrack approval from the United States Food and Drug Administration in October 1998 for use in the treatment of Crohn's disease. It is being marketed in Canada by Schering Canada, Inc, who is also a cosponsor of the specialist group responsible for the clinical practice guidelines appearing in this issue (pages 367-370). Centocor has listed the adverse reactions of intravenous infliximab in its product insert (for December 1, 2000) under the following headings: 'infusionrelated reactions', 'infections', 'autoantibodies/lupus-like syndrome', 'malignancies/lymphoproliferative disease' and 'other adverse reactions' (10). An update from the manufacturer February 15, 2001 provided no new cases of lymphoproliferative disease in treated patients (personal communication); however, the manufacturer has recently notified its investigators of a natural killer cell lymphoma and three deaths in 33 cases of tuberculosis after infliximab treatment (personal communications). Finally, a case of pneumocystis pneumonia was recently reported in a patient with Crohn's disease treated with infliximab, raising the spectre of an array of potential ubiquitous, but serious, opportunistic infections (11).

A guideline is defined in Webster's New World Dictionary of the American Language (12) as "...a standard or principle by which to make a judgement or determine a policy or course of action...". For the practising clinician, a set of guidelines may help in patient management. Indeed, in a recently published Canadian study, failure to follow guidelines for the treatment of asthma was shown to lead to negative consequences (13). Guidelines may be especially valuable in a condition such as Crohn's disease because treatment has traditionally been directed at controlling symptoms that have been attributed to disease activity and the inflammatory response. Clearly, if the cause and cure of Crohn's disease were known, treatment would presumably be more straightforward and clinical practice guidelines might not be required. One would simply treat and the disease would disappear. On the other hand, attempting to translate the results of a pharmaceutical trial into current clinical practice is no easy task, particularly because there may be many clinical situations not addressed by the drug trial (14). Adults with 'treatment-resistant' Crohn's disease who have received infliximab in clinical trials, for example, seniors, pregnant women and patients with disease in unusual disease locations (eg, jejunum, duodenum), have not been well represented (15). And, as most experienced clinicians appreciate in such a heterogeneous disorder, patients with anatomically similar disease in commonly detected sites can have vastly different presentations, severity of illness and disability. 
In the evaluation of a new treatment, particularly for a novel agent such as infliximab, there is a critical requirement to evaluate efficacy and the adverse event profile. Although a clinical trial may be scientifically well done, a treating physician must also be convinced that trial end points to assess efficacy reasonably reflect the 'real world' and must be comfortable that sufficient time has elapsed to enumerate adverse events accurately. Although these issues of efficacy and adverse effects will be considered further, it is worth reminding ourselves that the relative benefit of a new, compared with an old and more traditional, treatment must be evident. Otherwise, the rationale for abandonment of a traditional treatment may not be clear. For example, steroids, long used in the management of Crohn's disease, have only been evaluated in clinical trials, approximating the modern standard to a limited degree. Some adverse events in Crohn's disease, historically attributed to steroids, such as osteoporosis or osteonecrosis, may also be due to the disease per se (15-17). The clinician also needs to be satisfied that 'continuing symptoms' (to use the terminology in these practice guidelines) are due to the inflammatory process and not some other cause. Clearly, a lactose-free diet, use of an antidiarrheal agent, a bile-acid binder, avoidance of alcohol or treatment of an occult infection may be the correct approach, not another course of steroids, an increased dose or addition of another 'conventional' medication that modulates the immune response, or a novel biological agent such as infliximab. In published clinical trial data, an implicit assumption is made that such issues have been thoroughly addressed in each patient entered into the trial so that only assessment of efficacy and a tabulation of adverse events related to the specific study drug are needed. Finally, the interpretation of infliximab studies that have generated these practice guidelines is made more difficult because the study design permitted the use of 'conventional therapies', including steroids.

There are other poorly defined issues related to the modern randomized clinical trial that cannot be ignored, including infliximab studies. In some, including those (7-9) noted by the specialist group in this issue, the physicians who entered patients into trials differed from those who analyzed results and published the data. In part, this 'dissociation' may be essential to facilitate patient numbers for the trial, but spreading the 'action' around to multiple centres also serves as a useful marketing tool for the company sponsoring the trial. Moreover, investigators are well compensated in such studies, which may predispose those involved to favour a given product. Unfortunately, the influence, if any, of these factors on the 'purity' of a clinical trial in Crohn's disease has not been defined. For some journal publications (including this journal), the degree of investigator independence from the pharmaceutical firm may also be difficult to assess because author shareholders, honoraria or other stipends for 'research' may not be specified. No attempt is made to evaluate this issue here, but the motivation of the pharmaceutical industry and those involved in the development and marketing of a product, particularly a novel agent such as infliximab, may differ substantially from those of a clinician faced with a difficult patient.

For these practice guidelines, three randomized, controlled trials were cited by the committee as the best evidence for infliximab efficacy in the treatment of symptoms of Crohn's disease (7-9). Let me attempt to explore these with respect to end points used in the studies. Targan et al (7) reported a blinded and placebo-controlled dose-ranging study of infliximab (ie, 5, 10 and $20 \mathrm{mg} / \mathrm{kg}$ ) in 108 patients with a calculated Crohn's Disease Activity Index (CDAI) of 220 to 400 . This range of the CDAI is thought to generally approximate disease that might be seen in an ambulatory setting, not those with limited or mild symptoms or those sufficiently ill that hospitalization might be required. The selected placebo was intravenous albumin. Recruitment from multiple centres in different countries was done, including Canada (McMaster University, Hamilton, Ontario: two patients). Mesalamine, azathioprine, 6-mercaptopurine and steroids were permitted throughout the study in a 'stable' dose leading to the label of the 'treatment-resistant' patient. A clinical response, defined as a CDAI reduction of 70 points, was seen in 54 of $83(65 \%)$ treated patients at four weeks after infusion, but this number fell to only 34 of $83(41 \%)$ at 12 weeks. A clinical remission, defined as a CDAI less than 150 , occurred in $33 \%$ at four weeks, but data were no longer statistically significant at 12 weeks. Although the study was designed to demonstrate a dose-response, this was not achieved (an issue that still remains unresolved). Efficacy of infliximab was concluded in the short term for these CDAI-based definitions of response and remission. In a retreatment study by Rutgeerts et al (8) of a small number of patients infused repeatedly with $10 \mathrm{mg} / \mathrm{kg}$ of infliximab every eight weeks, maintenance of response (not remission) was maintained in $72 \%$ at week 36 . This statistical trend, however, was no longer evident eight weeks after the final infusion at week 44. Nevertheless, it was concluded that 'long term' treatment with infliximab showed efficacy in Crohn's disease. Unfortunately, as time has shown, the CDAI used in these two clinical trials may not be entirely useful as a guide for patient management. It is a highly subjective measure, even for experienced users in clinical trials, and remains prone to observer variation, and disease complications are heavily weighted by 'discriminant factors'. In an international study (18), for example, the same case history elicited different estimates of the CDAI, differing by up to 430 points among seven different experts! If a CDAI difference of 100 points (rather than 70), for example, had been used, would there still be a statistical difference in response rates? Similar conclusions regarding the limitations of such indexes and their lack of correlation with the colonoscopic and histological changes have also been noted (19). Geographic differences may also occur in multicentre studies for some components of the CDAI (18), an issue of particular relevance in these infliximab studies from North America and Europe. Critically, in both studies, there was no attempt to define the end points used in multiple centres by the clinicians 
entering patients to the study - estimates of intraobserver and interobserver variation were not provided, and arbitrary definitions of response and remission were defined only by historical reference (20).

For fistulous disease, Present et al (9) suggested that infused patients with infliximab in a dose of $5 \mathrm{mg} / \mathrm{kg}$ or $10 \mathrm{mg} / \mathrm{kg}$ effectively reduced drainage (defined as a reduction in drainage based on 'gentle' finger compression). 'Complete closure' for a period of a month occurred in 55\% of patients treated with $5 \mathrm{mg} / \mathrm{kg}$ and $38 \%$ of patients treated with $10 \mathrm{mg} / \mathrm{kg}$ (again, no dose response) compared with their placebo-treated patients of $13 \%$, seemingly not much different from treatments with different antibiotic regimens or uncontrolled studies reporting similar success with immunomodulatory agents such as methotrexate. In these studies, the end points of reduced drainage or even closure of a fistula are again arbitrary and clearly do not equate with persistent healing of a epithelial-lined fistulous tract between two anatomically separate tissues. Despite serious frustration with a difficult clinical problem that most clinicians have experienced in Crohn's disease, there remains room for skepticism about equating temporary reduction in drainage from a fistulous tract or a short period of fistula closure with true healing of a fistula.

Clearly, if infliximab satisfied concerns regarding efficacy in treatment of some of the symptoms and complications of the disease, such as fistulas, then adverse events, if limited, might convince the clinician that this novel ther-

\section{REFERENCES}

1. Van Dullemen HM, Van Deventer SJH, Hommes DW, et al. Treatment of Crohn's disease with anti-tumor necrosis factor chimeric monoclonal antibody (cA2). Gastroenterology 1995;109:129-35.

2. Siegel SA, Shealy DJ, Nakada MT, et al. The mouse/human chimeric monoclonal antibody cA2 neutralizes TNF in vitro and protects transgenic mice from cachexia and TNF lethality in vivo. Cytokine 1995; 7:15-25.

3. Scallon BJ, Moore MA, Trinh H, Knight DM, Ghrayeb J. Chimeric anti-TNF monoclonal antibody cA2 binds recombinant transmembrane TNF-alpha and activates immune effector functions. Cytokine 1995;7:251-9.

4. Braegger CP, Nicholls S, Murch SH, Stephens S, MacDonald TT. Tumor necrosis factor alpha in stool as a marker of intestinal inflammation. Lancet 1992;339:89-91.

5. MacDonald TT, Hutchings P, Choy M, Murch S, Cooke A. Tumour necrosis factor-alpha and interferon-gamma production measured at the single cell level in normal and inflamed human intestine. Clin Exp Immunol 1990;81:301-5.

6. Breese EJ, Michie CA, Nicholls SW, et al. Tumor necrosis factor alpha-producing cells in the intestinal mucosa of children with inflammatory bowel disease. Gastroenterology 1994;106:1455-66.

7. Targan SR, Hanauer SB, Van Deventer SJH, et al. A short-term study of chimeric monoclonal antibody cA2 to tumor necrosis factor alpha for Crohn's disease. Crohn's Disease cA2 Study Group. N Engl J Med 1997;337:1029-35.

8. Rutgeerts P, D'Haens G, Targan S, et al. Efficacy and safety of retreatment with anti-tumor necrosis factor antibody (infliximab) to maintain remission in Crohn's Disease. Gastroenterology 1999;117:761-9.

9. Present DH, Rutgeerts P, Targan S, et al. Infliximab for the treatment of fistulas in patients with Crohn's disease. N Engl J Med 1999;340:1398-405.

10. Remicade (infliximab recombinant) for IV injection product insert. Malvern: Centocor, Inc, Revised December 1, 2000. apy is worth pursuing. Common adverse reactions that have been recognized to date include hypersensitivity and infusion reactions. These have been generally mild, but rarely can be life-threatening. It has been associated with the production of antibodies to the foreign protein (termed human antichimeric antibodies) and lupus-like reactions. It has been associated with some infections, but only recently have more serious opportunistic infections been recognized. This will bear close observation because fatalities have been recorded and the manufacturer has warned the user that the agent should not be administered to those with a clinically important and active infection, as well as latent infections, such as tuberculosis. Finally, there are some realistic concerns regarding the longer term and the potential development of lymphoproliferative diseases. Data are limited on lymphoma in Crohn's disease (21), including those treated to date with infliximab (22). In Canada, there are also only very limited data (23), with only one of 877 patients developing a Hodgkin's type lymphoma, probably not much different from a similar age-matched population that does not have Crohn's disease. Because lymphoma may not be observed for several years, it is not possible to conclude that this will not be observed as a future complication of this novel therapy.

These are important times for patients with Crohn's disease, with research heavily focused on exploring novel treatment options. The wise clinician will be wary, cautious and wait for the right time to treat.

11. Panaccione R, Fraser K, Read R. Pneumocystis pneumonia following introduction of methotrexate and treatment with infliximab: cause for concern? Can J Gastroenterol 2001;15(Suppl A):51A.

12. Guralink DB, ed. Webster's New World Dictionary of the American Language, 2nd College edn. New York: Simon and Schuster, 1984.

13. Anis AH, Lynd LD, Wang X, et al. Double trouble: impact of inappropriate use of asthma medication on the use of health care resources. Can Med Assoc J 2001;164:625-31.

14. Sutherland LR. Principles of controlled trials in inflammatory bowel disease. In: Kirsner JB, ed. Inflammatory Bowel Disease, 5th edn. New York: WB Saunders, 2000:503-9.

15. Freeman HJ, Kwan WCP. Non-steroid associated osteonecrosis of the femoral heads in two patients with inflammatory bowel disease. N Engl J Med 1993;329:1314-6.

16. Freeman HJ. Osteomyelitis and osteonecrosis in inflammatory bowel disease. Can J Gastroenterol 1997;11:601-6.

17. Freeman HJ, Owen DA, Millan M. Granulomatous osteonecrosis in Crohn's disease. Can J Gastroenterol 2000;14:951-4.

18. De Dombal FT, Softley A. IOIBD report no 1: observer variation in calculating indices of severity and activity in Crohn's disease. Gut 1987;28:474-81.

19. Gomes P, Du Boulay C, Smith CL, Holdstock G. Relationship between disease activity indices and colonoscopic findings in patients with colonic inflammatory bowel disease. Gut 1986;27:92-5.

20. Best WR, Becktel JM, Singleton JW. Rederived values of the eight coefficients of the Crohn's Disease Activity Index (CDAI). Gastroenterology 1979;77:843-6.

21. Greenstein AJ, Gennuso R, Sachar DB, et al. Extraintestinal cancers in inflammatory bowel disease. Cancer 1985;56:2914-21.

22. Bickston SJ, Lichtenstein GR, Arseneau KO, Cohen RB, Cominelli F. The relationship between infliximab treatment and lymphoma in Crohn's Disease. Gastroenterology 1999;17:1433-7.

23. Freeman HJ. Colorectal cancer in Crohn's disease. Can J Gastroenterol (In press) 


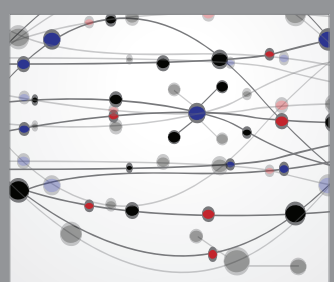

The Scientific World Journal
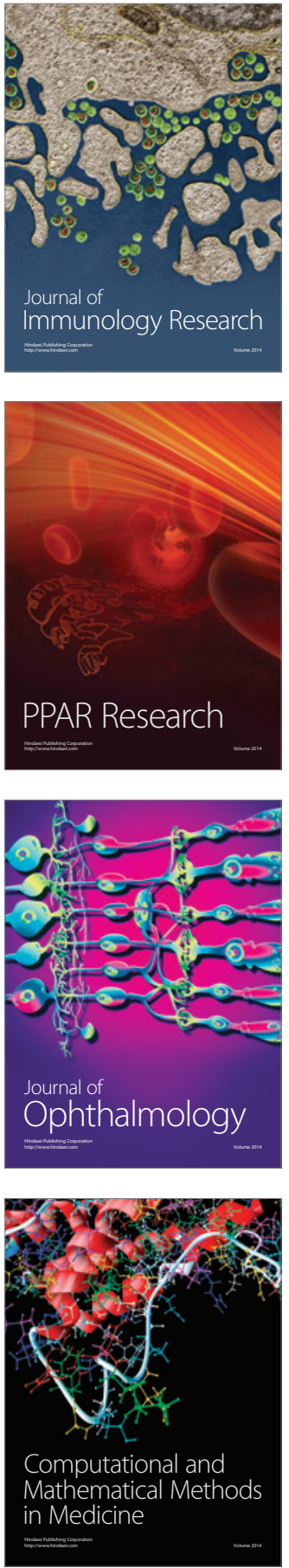

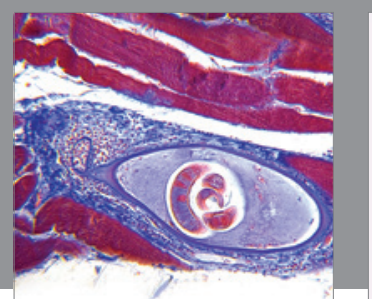

Gastroenterology Research and Practice

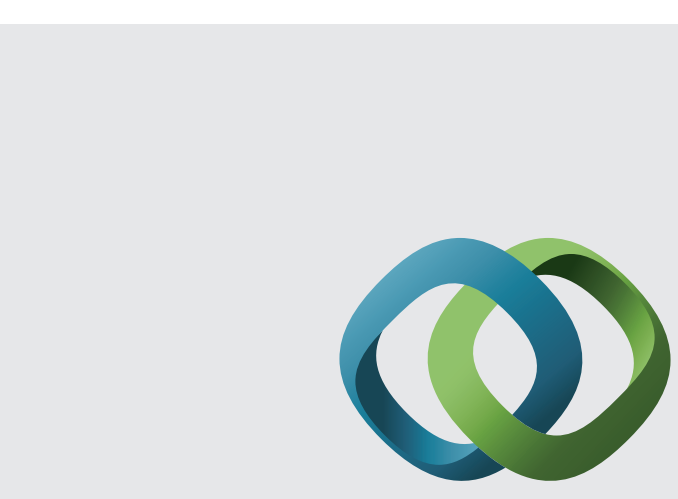

\section{Hindawi}

Submit your manuscripts at

http://www.hindawi.com
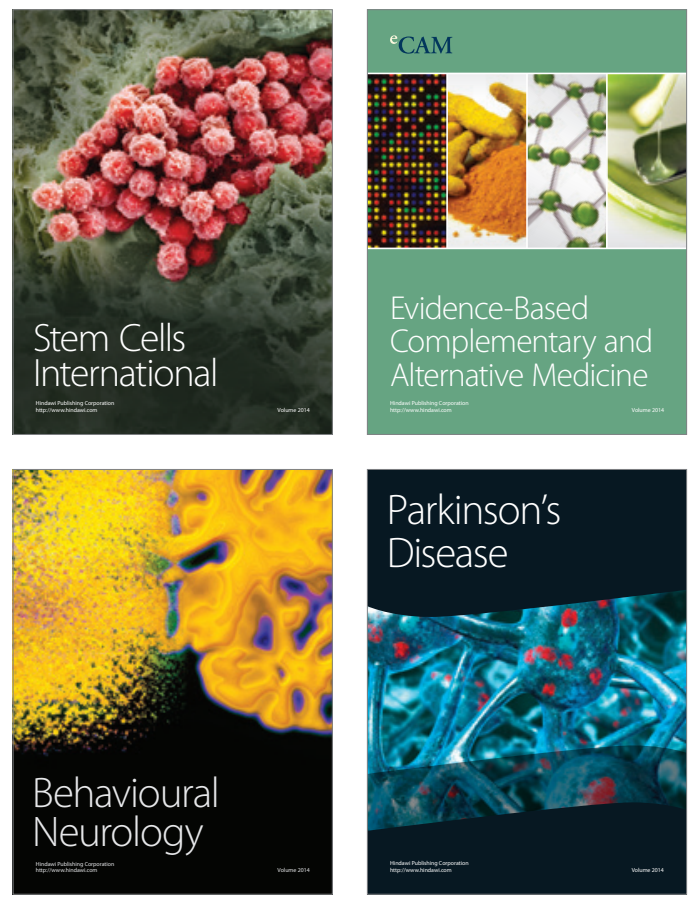
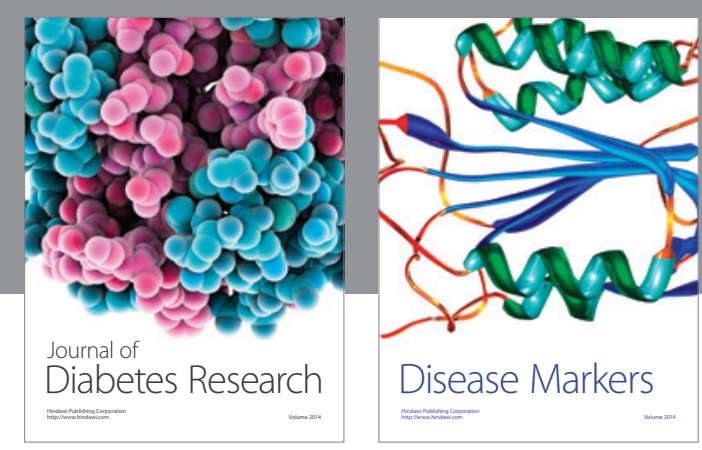

Disease Markers
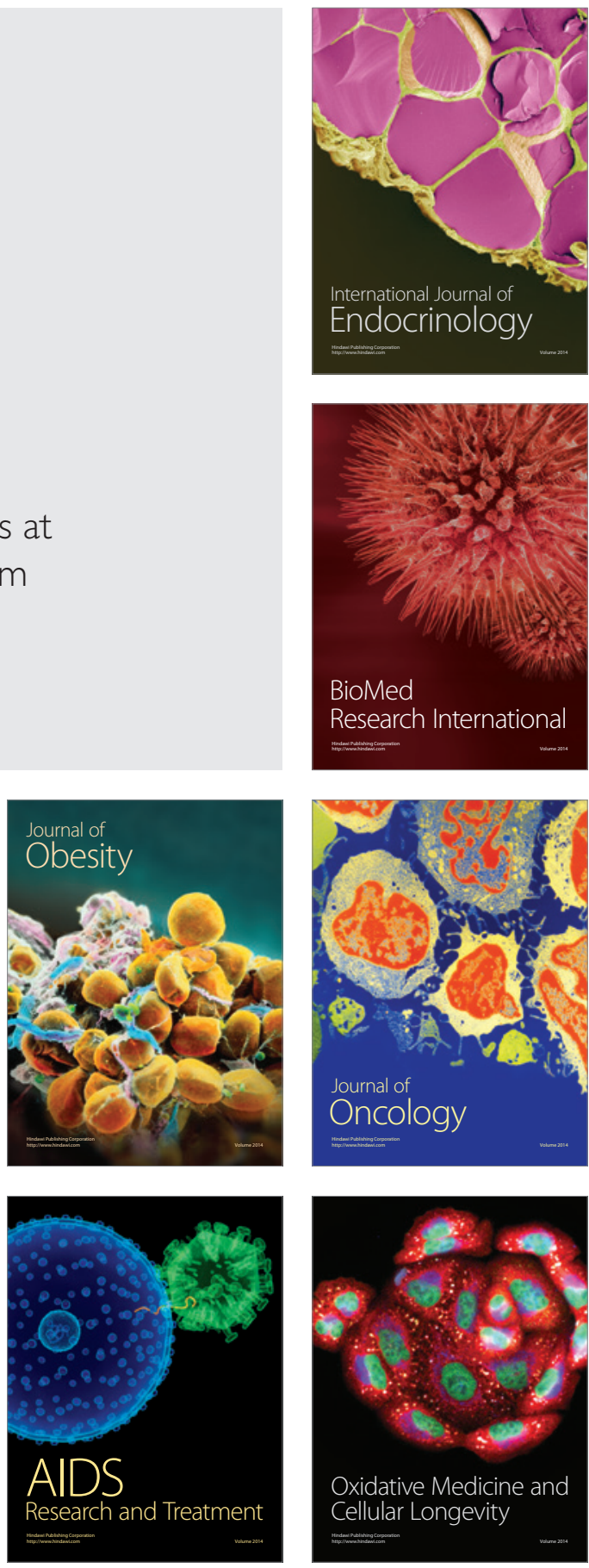